\title{
POSIBLES ABORDAJES DE TERAPIA OCUPACIONAL EN LA EDUCACIÓN SEXUAL DE NIÑOS, NIÑAS, ADOLESCENTES Y JÓVENES LESBIANAS, GAY, BISEXUALES Y TRANS: UNA REVISIÓN BIBLIOGRÁFICA
}

\author{
POSSIBLE APPROACHES TO OCCUPATIONAL THERAPY IN THE SEXUAL \\ EDUCATION OF CHILDREN, ADOLESCENTS AND YOUNG PEOPLE LESBIAN, GAY, \\ BISEXUAL AND TRANS: A BIBLIOGRAPHIC REVIEW
}

\section{Jose Ignacio Marchant Castillo'}

\begin{abstract}
RESUMEN
La sexualidad se presenta como un tabú desde la sociedad heteronormada, por lo que las implicancias, que la educación sexual, puede tener en las diversas áreas de ocupación de niños/as, adolescentes y jóvenes se han dejado de lado. Conllevando a situaciones de injusticias ocupacionales, que propiciarían un impacto negativo en el desarrollo y la salud mental de la población infanto-juvenil, a causa de maltratos por su orientación sexual, identidad y/o expresión de género en sus principales contextos de participación. Objetivo: Identificar y dar a conocer los posibles abordajes de terapia ocupacional en la educación sexual de niños/as, adolescentes y jóvenes lesbianas, gay, bisexuales y trans. Metodología: Se realiza una revisión bibliográfica narrativa en bases de datos científicas; Pubmed, Ebsco, psycinfo, Medline (Ovid), BEIC, OTseeker y Cochrane. Resultados y análisis: Se obtienen 120 artículos relacionados al tema, quedando 6, tras la aplicación de los criterios de inclusión y exclusión. Conclusión: Se pone de manifiesto la necesidad de poseer terapeutas ocupacionales en instituciones educativas, capacitados en el área sexual, pues promueven la justicia ocupacional, evitando dificultades que impactan en la salud mental y la calidad de vida de la población infanto-juvenil con diversidad sexual, por medio de intervenciones basadas en el interés, el dialogo, la reflexión y participación activa, centrándose en las necesidades reales de la población, integrando las nuevas tecnologías, con el fin de otorgarles ambientes físicos, sociales y virtuales significativos en los cuales aprender.
\end{abstract}

\section{PALABRAS CLAVES}

Niños, niñas y adolescentes, jóvenes, educación sexual, diversidad de Género, terapia ocupacional.

1 Licenciado en ciencias de la ocupación humana e integración Psicosocial, Terapeuta Ocupacional con mención en Rehabilitación Psicosocial, Universidad de los Andes. https://orcid.org/0000-0001-6449-6672. Código Postal: 8830332. To.josemarchant@gmail.com. Tel.: +56994157561. 


\begin{abstract}
Sexuality is presented as a taboo from heteronormal society, so the implications that sexual education can have in the various areas of occupation of children, adolescents and young people have been neglected. Leading to situations of occupational injustices, which would lead to a negative impact on the development and mental health of the child-youth population, due to abuse due to their sexual orientation, identity and / or gender expression in their main contexts of participation. Objective: Identify and publicize the possible approaches of occupational therapy in the sexual education of children, adolescents and young lesbians, gay, bisexual and trans. Methodology: A narrative bibliographic review is carried out in scientific databases; Pubmed, Ebsco, psycinfo, Medline (Ovid), BEIC, OTseeker and Cochrane. Results and analysis: 120 articles related to the subject are obtained, leaving 6, after the application of the inclusion and exclusion criteria. Conclusion: The need to have occupational therapists in educational institutions trained in the sexual area is highlighted, as they promote occupational justice, avoiding difficulties that impact on the mental health and quality of life of the child-youth population with sexual diversity, through interventions based on interest, dialogue, reflection and active participation, focusing on the real needs of the population, integrating new technologies, in order to grant them significant physical, social and virtual environments in which to learn.
\end{abstract}

\title{
KEYWORDS
}

Children and adolescents, young people, sex education, gender diversity, occupational therapy.

Recibido: 29 Mayo 2019

Aceptado: 27 Noviembre 2019

\section{INTRODUCCIÓN}

La sexualidad es un proceso inherente a lo largo de la vida de las personas (Cerruti, 2008), y un componente esencial en el cuidado holístico de la salud (Jones, Weerakoon \& Pynor, 2005), independiente de su condición, y del cual, debiese instruirse durante cada etapa del ciclo vital, con el fin de evitar enfermedades de transmisión sexual, uso inadecuado de anticonceptivos, embarazos adolescentes (Moral de la Rubia, 2007) e intentos de suicidios, particularmente de personas transexuales (Kano, 2016; Torres, 2015; Bazargan, 2012; Poteat, 2013). Si bien, en Chile no existe una ley que regule una educación sexual integral, existe la Ley $N^{0} 20.418$ sobre información, orientación y prestaciones en materia de regulación de la fertilidad, que está en proceso de modificación, con el fin de facilitar el acceso a los conocimientos desde quinto básico. Que junto al Ministerio de educación y a su política de convivencia escolar, buscan garantizar un enfoque inclusivo y de derechos en los colegios, que propicie una cultura hacia el reconocimiento e incorporación de la diversidad en los procesos educativos.

Dentro de esta diversidad social, encontramos personas cuya expresión de género, orientación sexual y/o sexo biológico no cumplen las demandas sociales de la cultura heteronormada, que dicta que la heterosexualidad y los estereotipos de género son lo ideal, apreciando al hombre heterosexual como un ser masculino, en lo alto de una jerarquía, por sobre la mujer femenina y otros hombres homosexuales (Galaz \& Troncoso, 2016, p. 99).

Desde terapia ocupacional (TO), se comprende que las personas con una diversidad sexual, en cuanto a identidad, expresión y/u orientación sexual pueden ver restringido, desde el ambiente social, el acceso a una participación digna y significativa en las ocupaciones de la vida diaria y en sus elecciones ocupacionales, estableciéndose una situación de apartheid ocupacional (Kronenberg,Pollard y Simó, 2006) e injusticia ocupacional (Wilcock, 2006). Por lo que es atingente que esta población esté en los abordajes de TO, intentando incluir todos los intereses e inquietudes posibles para realizar intervenciones satisfactorias, incluidas las actitudes, conductas y prácticas influidas de la motivación por vivenciar y expresar la sexualidad, practicando y adquiriendo acciones observables, concretas y dirigidas, que contienen a las habilidades motoras, de procesamiento y las habilidades de interacción y comunicación (Kielhofner, 2011), de manera específica durante cada etapa del ciclo vital y, que se observan en aquellas actividades relacionadas a la sexualidad dentro de los hábitos de rutina, desempeño y estilo (De las Heras, 2015. p. 32).

Esta "limitación" en la participación de niños y niñas, adolescentes y jóvenes (NNAJ) lesbianas, gays, bisexuales 
y trans (LGBT) en el contexto escolar se genera por experimentar frecuentes situaciones de maltratos físicos y/o verbales por parte de los pares e incluso profesionales de la educación (Kosciw, Greytak, Zongrone, Clark \& Truong, 2018), simplemente por diferenciarse de la "norma". Se describe que el $75 \%$ de los estudiantes encuestados fue testigo de violencia escolary, entre el 40-45\% habían sido participes de dicha violencia, en relación a esto, se afirma que las diversidades sexuales sufren con mayor intensidad las agresiones (Cáceres, Silva-Santisteban, Salazar, Cuadros, Olivos, \& Segura, 2011. Citado en UNESCO, 2015). Si bien en Chile no existen estadísticas concretas en relación a esta situación, posiblemente por el estigma que conlleva el identificarse como "persona diversa", en países como México, la violencia hacia los escolares LGBT, alcanzaría el $67 \%$ (Youth Coalition, cojess México \& Eneache, 2012). Estas experiencias negativas conllevarían a una disminución de la participación ocupacional en el contexto educativo, viéndose en alza el ausentismo, por el clima escolar hostil, impactando directamente en el desempeño académico y en el desarrollo de la identidad de NNAJ, pues según Slesaransky-Poe (2013), "los climas escolares hostiles afectan no sólo el rendimiento académico, sino también el desarrollo social, emocional y psicológico" (p. 2). Traduciéndose en una escasa red de apoyo y menores oportunidades a futuro, en el acceso al área laboral (Drydakis, 2019) produciendo y reproduciendo contextos de injusticia ocupacional.

Como, el contexto escolar es el ambiente donde los NNAJ pasan la mayor parte del día y donde se desarroIlan a diario, es importante mencionar que; el no poseer apoyo, tanto de la red familiar como de la red educativa, podría contribuir en bajos niveles de autoestima, lo que afecta de manera crítica a los jóvenes, pues aumenta la probabilidad de presentar problemas de salud mental (Kann, 2011), como la depresión y la ansiedad (Movement Advancement Project, 2012, p. 3), llevándolos al aislamiento y en muchos casos, a ver el suicidio como única solución (Cáceres \& Salazar, 2012; Fundación todo mejora, 2017; Kosciw et al, 2018), por lo que podríamos decir que, la población LGBT presentan un riesgo mayor de intentos de suicidio, 4 veces más alto que sus pares heterosexuales (Grossman \& D'augelli, 2007), mientras que en aquellos que cuestionan su sexualidad, el riesgo es 3 veces mayor que sus pares que no realizan tal cuestionamiento (Kann, 2011; Meyer, 2003. Citado en León, Chaigneau, del Río, \& Rodríguez, 2016). Además, presentan con mayor frecuencia adicción a drogas y alcohol, y, por consiguiente, mayor tendencia a comportamientos sexuales de alto riesgo (Hillier, Turner, Mitchell, 2005) como el inicio precoz de las prácticas sexuales, el uso inadecuado de anti-conceptivos y la tenencia de múltiples parejas sexuales (Gökengin et al. 2003. Citado en Yubero, Larrañaga y Yubero, 2013).

Es por estas dificultades psicosociales que es importante conocer si existen y cuáles son los abordajes desde terapia ocupacional en educación sexual de la población infanto-juvenil con diversidad sexual.

\section{MÉTOdo}

La metodología empleada corresponde a la revisión bibliográfica narrativa (Aguilera, 2014), en la cual se realizaron búsquedas sistemáticas en las bases de datos Pubmed, Ebsco, psycinfo, Medline (Ovid), BEIC, OTseeker, Cochrane. Una búsqueda complementaria en Google Schoolar y el portal de la revista chilena de terapia ocupacional, por medio de un computador con conexión a wifi, que poseía los programas Word y Acrobat Reader DC.

Los criterios de inclusión utilizados para la selección de artículos científicos y documentos académicos fueron: Artículos que hablen de educación sexual en niños y niñas, jóvenes y adolescentes LGBT, de abordajes de terapia ocupacional en esta población y los beneficios que las intervenciones pueden generar en la prevención y/o disminución de problemas de salud mental y/o sexual, el impacto en los ambientes familiares y educacionales. Artículos cualitativos y/o cuantitativos, en idioma español e inglés que sumen 40 puntos o más en el test de Craap (Blakeslee, 2004).

Excluyendo así los artículos que no sean accesibles de forma gratuita, textos incompletos, archivos previos al año 2007 y artículos que consideren a los jóvenes por sobre los 25 años de edad. Se presenta la tabla PIO (Tabla 1), de confección personal para presentar los términos buscados en ésta revisión bibliográfica. 
TABLA 1

DESCRIPCIÓN ESPECIFICA DE LOS CONCEPTOS INVESTIGADOS POR EL AUTOR

\begin{tabular}{ll}
\hline Persona & Niños y niñas, adolescentes y jóvenes LGBT \\
\hline Intervención & Educación sexual \\
\hline Resultados & $\begin{array}{l}\text { Tipos de abordajes de terapia ocupacional, que buscan el beneficio de niños y } \\
\text { niñas, jóvenes y adolescentes LGBT, disminuyendo la probabilidad de presentar } \\
\text { problemas de salud mental y sexual, apoyar la no deserción escolar y la acepta- } \\
\text { ción familiar, mejorando las interacciones sociales dentro del ambiente familiary } \\
\text { escolar }\end{array}$ \\
\hline
\end{tabular}

Conceptos buscados en las diversas bases de datos y buscadores; cabe mencionar que éstos términos fueron buscados en español y en inglés para obtener la mayor cantidad de documentos relacionados al tema.

\section{FLUJOGRAMA DE LA REVISIÓN BIBLIOGRÁFICA}

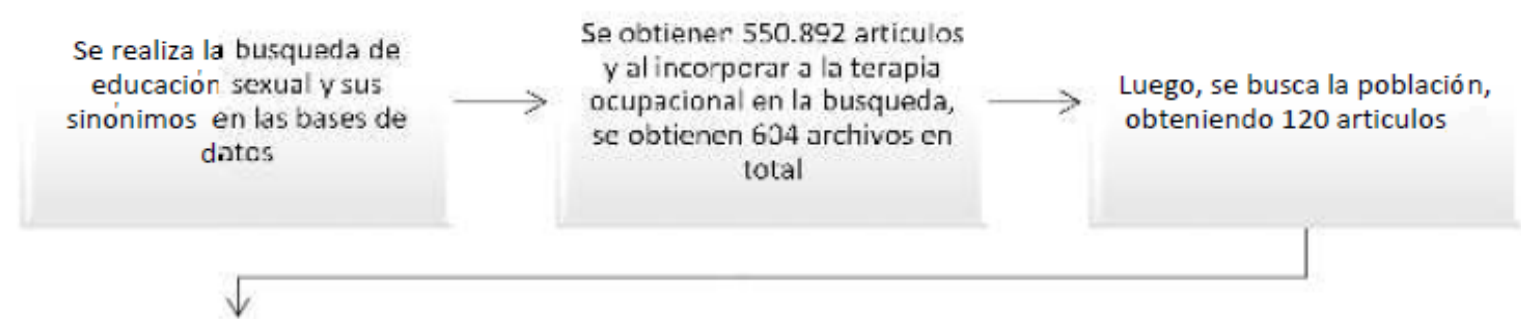

Al realizar la busqueda de Girl o boy se observa la redireccion a documentos donde aparece el termino woman y man por lo que no se consideran en la busqueda

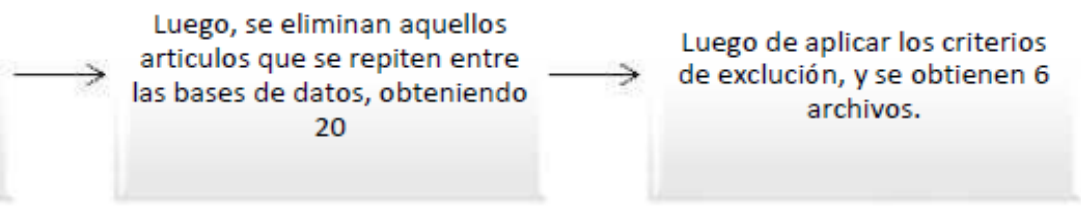

\section{Resultados}

Si bien en la búsqueda sistemática se hallaron 120 documentos que abarcaban los conceptos descritos en los criterios de inclusión. Se observa una deuda en cuanto a la actualización del conocimiento, pues el levantamiento de información desde los abordajes de terapia ocupacional en la educación sexual en población infantojuvenil con diversidad sexual generó un escaso pool de 6 opciones para desarrollar éste documento. Donde la mayor parte de los archivos hallados, en primera instancia, son antiguos y desactualizados, como se concluye en el estudio de Monzeli \& Lopes (2012), concordando con lo planteado por Pedraza (2014) en relación a que la investigación en TO escasamente aborda la sexualidad, siendo esta la principal limitación del presente estudio.
Se logró apreciar el naciente y tímido interés por parte de los terapeutas ocupacionales (TOc) respecto al área sexual, pues la reconocen dentro de un todo que conforma a la persona y que cuyo abordaje permitirá intervenir de forma integral y holística a ésta, reflejándose en el Marco de trabajo de la American Occupational Therapy Association (2008), donde reconocen la actividad sexual como una ocupación básica de la vida diaria. Aunque es incipiente el interés por la sexualidad, no cabe duda que desde lo teórico posemos una base bastante sólida, para poder comenzar a participar en dicha área (Rueda, 2005). Sin embargo, dicha participación se ve limitada por varios factores, que se describen en la publicación de Mc Grath \& Sakallariau (2016), y que impactarían en la praxis habitual, donde cabe destacar que, aquel factor que puede estar influenciado con 
mayor fuerza, está dado por la escasa atención que se le da a esta área en el proceso de pre-grado (Hyland \& McGrath, 2013; McGrath \& Lynch, 2014) y en las escasas capacitaciones existentes en el área (Monzeli \& Lopes, 2012). Otro factor importante es que las instituciones de salud suelen tener estrictas políticas institucionales en relación a la sexualidad, y una modalidad transitoria, lo que no permitiría generar un vínculo con el usuario para que este pueda, gracias a los modos facilitadores que manejamos como TOc (Taylor, 2008. Citado en Bellido \& Berrueta, 2010), poder informarnos respecto a sus dificultades y/o limitaciones en el ejercicio pleno de su sexualidad (Couldrick, 1998, Hyland \& McGrath, 2013; McGrath \& Lynch, 2014; Penna y Sheehy, 2000. Citado en Mc Grath, \& Sakallariau, 2016), que pudiesen generar una desadaptación ocupacional entre lo que son, como y con quien expresan su sexualidad, versus quienes por norma tienen que ser, como y hacia quienes tienen que expresar su afectividad.

Es importante seguir generando información respecto a este tema para poder facilitar el proceso de las futuras generaciones de profesionales, evitándoles la inseguridad del cómo desempeñarse frente éstas situaciones (Crespillo \& Barbero, 2016), fomentando nuestra identidad como profesionales, capaces de poder participar de forma satisfactoria en los procesos en los que se requiera tratar temas de orientación sexual, limitaciones y/o restricciones en la expresión de género, mediación en relaciones con familiares, profesionales de la educación y los pares, otorgando ambientes facilitadores de la participación ocupacional en actividades lúdicas, significativas y centradas en los intereses de cada persona, como se menciona en el estudio de Ponzetti, Selman, Munro, Esmail, \& Adams (2009) y que incluyan las tecnologías actuales, como juegos virtuales (Gontijo, de Sena e Vasconcelos, Monteiro, Facundes, de Fátima, \& de Lima, 2016). Dichos abordajes, desde una perspectiva participativa, y con un moderador, que facilite el clima del grupo social (De las Heras, 2015) generarían resultados favorecedores inmediatamente al terminar la intervención, según lo descrito en el estudio de Gontijo et al (2016). Estos resultados se podrían traducir en una mejoría en el desempeño ocupacional en el contexto educativo, pues existiría menor ausentismo y deserción escolar, demostrándole a la sociedad educativa que los TOc son esenciales en sus dispositivos y en los procesos de educación sexual en todos los participantes y no tan solo a la población LGBT, pues como se mencionó anteriormente, todos poseemos esta faceta, pues es central y transversal para cualquier persona. Y, que irá definiendo la identidad personal, vocacional, ocupacional y sexual, integrando en dicho proceso las dimensiones biológica, social y motivacional (León, et al. 2016). Y, por lo que, debiesen construirse bajo un marco de justicia ocupacional, por medio de elecciones ocupacionales libres, sin restricciones ni barreras impuestas por normas absurdas de la sociedad.

\section{Discusión}

Gracias a este estudio, podemos reflexionar entorno a la propia práctica profesional y en relación a nuestro rol a la importancia que le damos al área de la sexualidad, y la inminente necesidad de capacitarnos, no tan solo en procedimientos clínicos, sino que también en aspectos históricos, culturales y políticos (Monzeli \& Lopes, 2012) que atañen a la sexualidad, que, sin duda infieren en la participación y el desempeño de los/as NNAJ LGBT que se desarrollan, muchas veces, en ambientes hostiles y lleno de injusticias ocupacionales. Pues, como profesionales socio-sanitarios y específicamente como TOc, buscamos, entre varios objetivos, evitar los ambientes limitadores de la participación ocupacional y los procesos de desadaptación ocupacional entre la identidad y competencia ocupacional (De las Heras, 2015. p33) de todos aquellos que exploran y vivencian la sexualidad, tratando de disminuir los factores de riesgo asociados a los problemas de salud mental (Sánchez, Polonio \& Pellegrini, 2013 p. 36), que puedan contribuir a desarrollar riesgos de autolesiones y suicidios (Movement Advancement Project, 2012. p. 3), la promoción de hábitos y rutinas saludables, la participación en contextos sociales y la mantención de una red de apoyo cercana, acorde a las necesidades y a las demandas tecnológicas de los NNAJ.

Además, para evitar el riesgo en cuanto a sintomatología o conducta de riesgo, es importante visibilizar, sensibilizar, educar respecto a la diversidad sexual y las expresiones de estas, normalizando la condición e integrándola a la cultura, no tan solo a las comunidades educativas y a los NNAJ, sino también a los padres, dándole nuevas acepciones a los roles que desempeñamos y los objetos que utilizamos dentro de las rutinas, lo que facilitará la participación y el desempeño ocupacional óptimo de los NNAJ en los diversos ambientes significativos en los que, por su ciclo vital debiesen, por derecho, acceder y participar de forma plena, segura y digna. 
Particularmente como TOc, no me gustaría encontrar adultos frustrados, que desperdicien su talento y su vida en ocupaciones y profesiones que no representan quienes son, pero que absurdamente, responden a estereotipos de su sexo biológico (Monteiro, Gontijo, Facundes \& Sena, 2015). Más bien, deseo que estos individuos tengan la oportunidad de desplegar y reafirmar su identidad ocupacional en base a los valores positivos que les entreguen los profesionales de la salud y la educación, junto a padres facilitadores (Ehrensaft, 2007), basados en el conocimiento, el amor y la sabiduría.

\section{REFERENCIAS BibLIOGRÁFICAS}

Aguilera Eguía, R. (2014). ¿ Revisión sistemática, revisión narrativa o metaanálisis. Revista de la Sociedad Española del Dolor, 27(6), 359-360.

American Occupational Therapy Association - AOTA. (2008). Occupational Therapy Practice Framework: Domain and process. The American Journal of Occupational Therapy.

Bazargan, M., \& Galvan, F. (2012). Perceived discrimination and depression among low-income Latina male-to-female transgender women. BMC public health, 12(1), 663.

Bellido Mainar J. R., Berrueta Maeztu L. M. (2010). La Relación Terapéutica en Terapia Ocupacional. TOG (A Coruña) -]; 8(13): [20p.]. Rescatado de: http://www.revistatog.com/num13/pdfs/ modelotaylor1.pdf

Blakeslee, S. (2010). Evaluating information: Applying the CRAAP test. Meriam Library, California State University, Chico.

Cáceres, C. F., \& Salazar, X. (2012). "Era como ir todos los días al matadero...": el bullying homofóbico en instituciones educativas públicas de Chile, Guatemala y Perú.

Cáceres, C., Silva-Santisteban, A., Salazar, X., Cuadros, J., Olivos, F., \& Segura, E. (2011). Estudio a través de Internet sobre «Bullying», $y$ sus manifestaciones homofóbicas en escuelas de Chile, Guatemala, México y Perú, y su impacto en la salud de jóvenes varones entre 18 y 24 años. Informe Final. Instituto de Estudios en Salud, Sexualidad y Desarrollo, Lima.

Cerruti, S. (2008). Sexualidad humana. Montevideo: OPS; 1992. (p. 24).

Couldrick, L. (1998). Sexual issues: An area of concern for occupational therapists?. British Journal of Occupational Therapy, 67(11), 493-496.

Couldrick, L. (2005). Sexual Expression and Occupational Therapy. British Journal of Occupational Therapy, 68(7), 315-318.

Crespillo, M. A., \& Barbero, Ó. G. (2016). Barreras en la Asistencia Sociosanitaria a Personas Transexuales: Una Revisión Sistemática de Metodología Cualitativa.

De las Heras, C. G. (2015). Desarrollo de integración de la teoría y la práctica del MOHO. Modelo de ocupación Humana (pp. 32-33). España: Síntesis.
Drydakis, N. (2019). Sexual orientation and labor market outcomes. IZA World of Labor.

Ehrensaft, D. (2007). Raising girlyboys: A parent's perspective. Studies in Gender and Sexuality, 8(3), 269-302.

Fundación Todo Mejora (2017). Manual de apoyo a profesores, tutores y apoderados; para enseñar sobre diversidad, orientación sexual e identidad y expresión de género. P. 8.

Galaz, Caterine, Troncoso, Lelya, \& Morrison, Rodolfo. (2016). Critical Views on Educational Intervention in Sexual Diversity. Revista Latinoamericana de educación inclusiva, 10(2), 93-111. https:// dx.doi.org/10.4067/S0718-73782016000200007

Gontijo, D. T., de Sena e Vasconcelos, A. C., Monteiro, R.J. S., Facundes, V.L.D., de Fátima Cordeiro Trajano, M., \& de Lima, L.S. (2016). Occupational Therapy and Sexual and Reproductive Health Promotion in Adolescence: A Case Study. Occupational therapy international, 23(1), 19-28.

Grossman, A. H., \& D'augelli, A. R. (2007). Transgender youth and life-threatening behaviors. Suicide and Life-Threatening Behavior, 37(5), 527-537.

Hillier, L., Mitchell, A., \& Turner, A. (2005). Writing themselves in again: 6 years on: The 2nd national report on the sexuality, health \& wellbeing of same-sex attracted young people. Australian Research Centre in Sex, Health and Society.

Hyland, A., \& Mc Grath, M. (2013). Sexuality and occupational therapy in Ireland-a case of ambivalence?. Disability and rehabilitation, 35(1), 73-80.

Jones, M. K., Weerakoon, P., \& Pynor, R. A. (2005). Survey of occupational therapy students' attitudes towards sexual issues in clinical practice. Occupational Therapy International, 12(2), 95-106.

Kann L. (2011). Sexual identity, sex of sexual contacts, and health-risk behaviors among students in grades 9-12: Youth risk behavior surveillance, selected sites, United States, 2001-2009. DIANE Publishing, 60(7), 2-51.

Kann, L., Kinchen, S., Shanklin, S. L., Flint, K. H., Hawkins, J., Harris, W.A.,... \& Whittle, L. (2014). Youth risk behavior surveillance-United States, 2013. Morbidity and Mortality Weekly Report: Surveillance Summaries, 63(4), 1-168.

Kano, M., Silva-Bañuelos, A. R., Sturm, R., \& Willging, C. E. (2016). Stakeholders' recommendations to improve patient-centered "LGBTQ" primary care in rural and multicultural practices. The Journal of the American Board of Family Medicine, 29(1), 156-160.

Kielhofner, G. (2011). Introducción al modelo de ocupación humana. Kielhofner G. Modelo de Ocupación Humana: Teoría y aplicación. $4^{a}$ ed. Madrid: Editorial Médica Panamericana, 1-7.

Kosciw, J. G., Greytak, E. A., Zongrone, A. D., Clark, C. M., \& Truong, N. L. (2018). The 2017 National School Climate Survey: The Experiences of Lesbian, Gay, Bisexual, Transgender, and Queer Youth in Our Nation's Schools. Gay, Lesbian and Straight Education Network (GLSEN). 121 West 27th Street Suite 804, New York, NY 10001. 
Kronenberg, F., Algado, S. S., \& Pollard, N. (2007). Terapia Ocupacional sin Fronteras: Aprendiendo del espíritu de supervivientes. Ed. Médica Panamericana.

Martínez, M. (2017). Apartheid Ocupacional de las diversidades trans: Importancia y desafío para la Terapia Ocupacional Social. Polyphōnía. Revista de Educación Inclusiva/Polyphōnía. Journal of Inclusive Education, 7(2).

McGrath, M., \& Lynch, E. (2014). Occupational therapists' perspectives on addressing sexual concerns of older adults in the context of rehabilitation. Disability and rehabilitation, 36(8), 651-657.

Mc Grath, M., \& Sakellariou, D. (2016). Why has so little progress been made in the practice of occupational therapy in relation to sexuality?. American Journal of Occupational Therapy, 70(1), 7001360010p1-7001360010p5.

Meyer, I. H. (2003). Prejudice, social stress, and mental health in lesbian, gay, and bisexual populations: conceptual issues and research evidence. Psychological bulletin, 129(5), 674-697. doi: 10.1037/0033-2909.129.5.674

Monteiro, R. J. S., Gontijo, D. T., Facundes, V. L. D., \& Sena, A. C. (2015). "Pensando como um menino é mais fácil": construções sobre as relações de gênero no discurso de meninas adolescentes. Revista de Terapia Ocupacional da Universidade de São Paulo, 26(2), 207-215.

Monzeli, G., \& Lopes, R. E. (2012). Terapia ocupacional e sexualidade: uma revisão nos periódicos nacionais e internacionais da área. Revista de Terapia Ocupacional da Universidade de São Paulo, 23(3), 237-244.

Moral de la Rubia, J. (2007). Conducta sexual y uso del preservativo en estudiantes universitarios. Medicina universitaria, 9(37), 173-180

Movement Advancement Project. (2012). Talking about suicide \& LGBT populations. Rescatado de : http://www.lgbtmap.org/ file/talking-about-suicide-and-lgbt-populations-2nd-edition.pdf

León, M. J., Chaigneau, S., del Río, P., \& Rodríguez, J. (2016). Sintomatología clínica en adolescentes chilenas heterosexuales y homosexuales. $\operatorname{Rev} G P U, 12(4), 413-9$.

Pedraza, T. (2014). Vejez y sexualidad: reflexiones para la práctica de terapia ocupacional. Revista chilena de terapia ocupacional, 14(2), 245-255.

Ponzetti Jr, J.J., Selman, J., Munro, B., Esmail, S., \& Adams, G. (2009). The effectiveness of participatory theatre with early adolescents in school-based sexuality education. Sex education, 9(1), 93-103.

Poteat, T., German, D., \& Kerrigan, D. (2013). Managing uncertainty: a grounded theory of stigma in transgender health care encounters. Social science \& medicine, 84, 22-29.

Rueda, L. Rueda, J. (2005) “El Enfoque Comunicacional en la formación de Terapeutas Ocupacionales: Aspectos teóricos y propuestas de aplicación." Revista Chilena de Terapia Ocupacional. №5.

Sánchez O., Polonio B., \& Pellegrini M. (2013). Terapia ocupacional en salud mental; Teoría y técnicas para la autonomía personal. Madrid; Editorial Médica Panamericana, S. A.
Sheehy, S. P. K. (2000). Sex education and schizophrenia: should occupational therapists offer sex education to people with schizophrenia?. Scandinavian Journal of Occupational Therapy, 7(3), 126-131.

Slesaransky-Poe, G. (2013). Interrumpiendo el discurso y la práctica género-y hetero- normativxs en las escuelas. In IIIJornadas CINIG de Estudios de Género y Feminismos, La Plata, 2013.

Taylor, R. R. (2008). The intentional relationship: Outpatient therapyand use of self. F. A. Davis.

Torres, C. G., Renfrew, M., Kenst, K., Tan-McGrory, A., Betancourt, J. R., \& López, L. (2015). Improving transgender health by building safe clinical environments that promote existing resilience: Results from a qualitative analysis of providers. BMC pediatrics, 15(1), 187.

UNESCO. (2015). La violencia homofóbica y transfóbica en el ámbito escolar: hacia centros educativos inclusivos y seguros en América Latina.

Wilcock, A. A. (2006). An occupational perspective of health. Slack Incorporated.

Youth Coalition, COJESS México y eneache. (2012). Primera encuesta nacional sobre bullying homofóbico. Rescatado de: http://www. enehache.com/EncuestaBullying/Bullying\%20homofobico-1(14May-12).pdf

Yubero, M., Larrañaga, E., \& Yubero, S. (2014). Actitudes y comportamiento sexual de riesgo de embarazo en jóvenes universitarios. Revista de Psicologia da Criança e do Adolescente, 4(2), 11-29.

\section{Anexo}

Se presentan la tabla Matriz con los documentos que cumplían los Criterios de Inclusión y exclusión. 


\begin{tabular}{|c|c|c|c|c|}
\hline 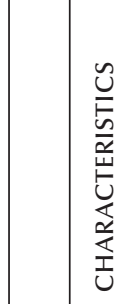 & 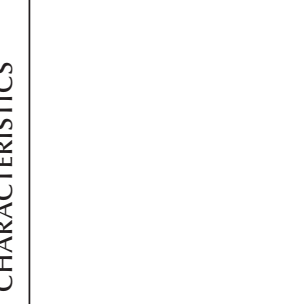 & 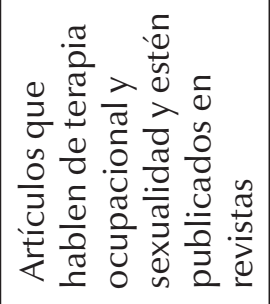 & 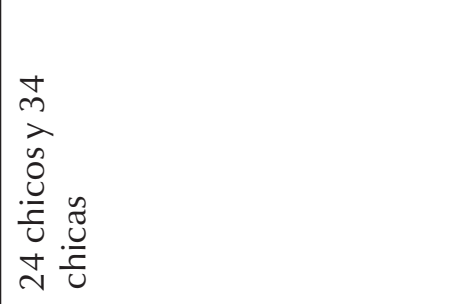 & 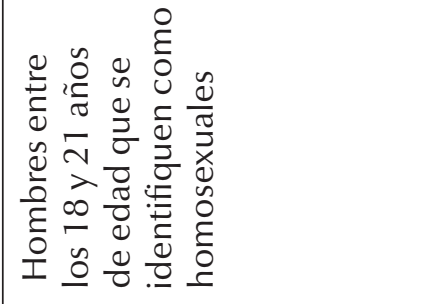 \\
\hline  & 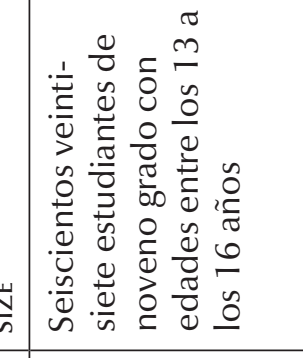 & & 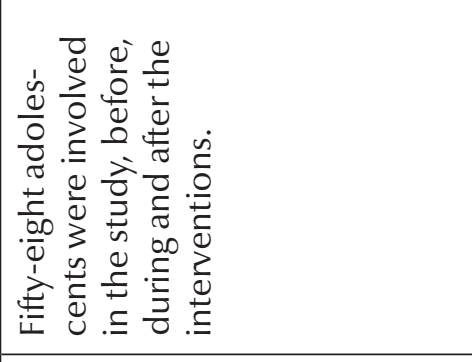 &  \\
\hline  &  & & 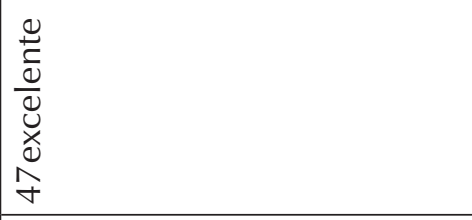 & 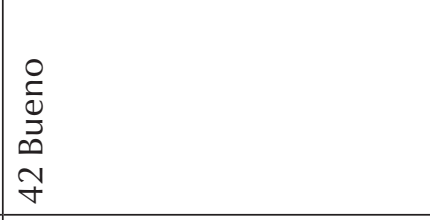 \\
\hline 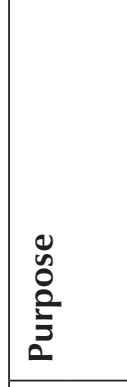 &  &  & 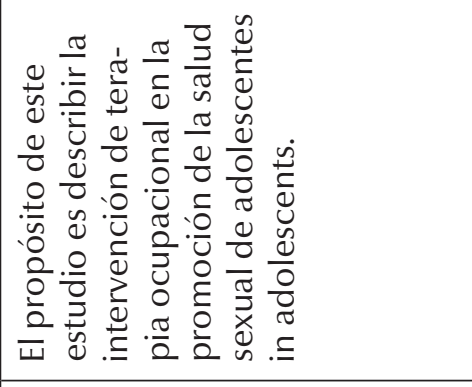 & 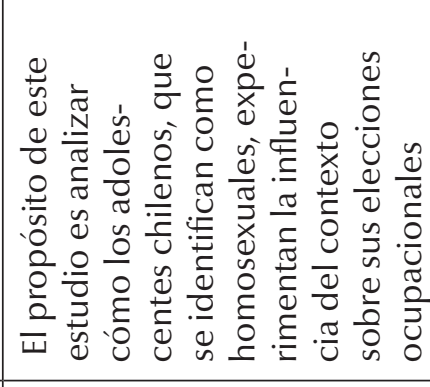 \\
\hline  & $\begin{array}{l}\text { Qे } \\
\text { iv }\end{array}$ & ㄱ & Ln & 늠 \\
\hline$\stackrel{0}{E}$ & 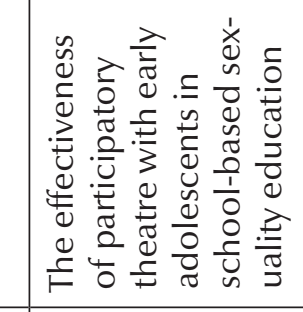 &  & 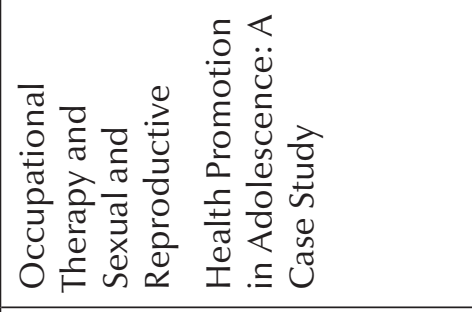 &  \\
\hline 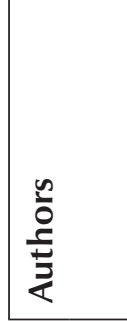 &  & 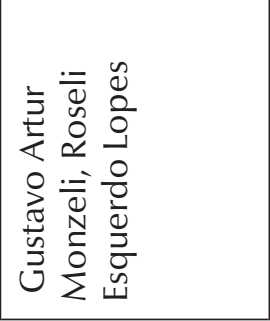 & 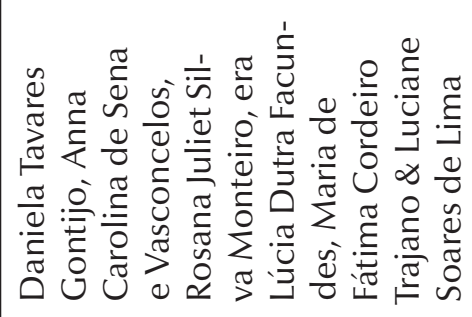 & 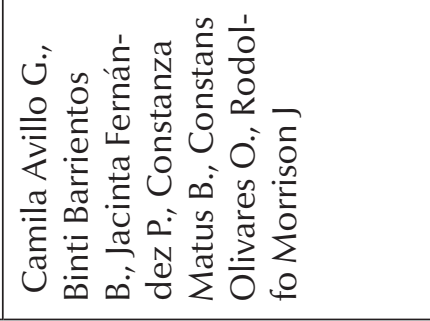 \\
\hline
\end{tabular}




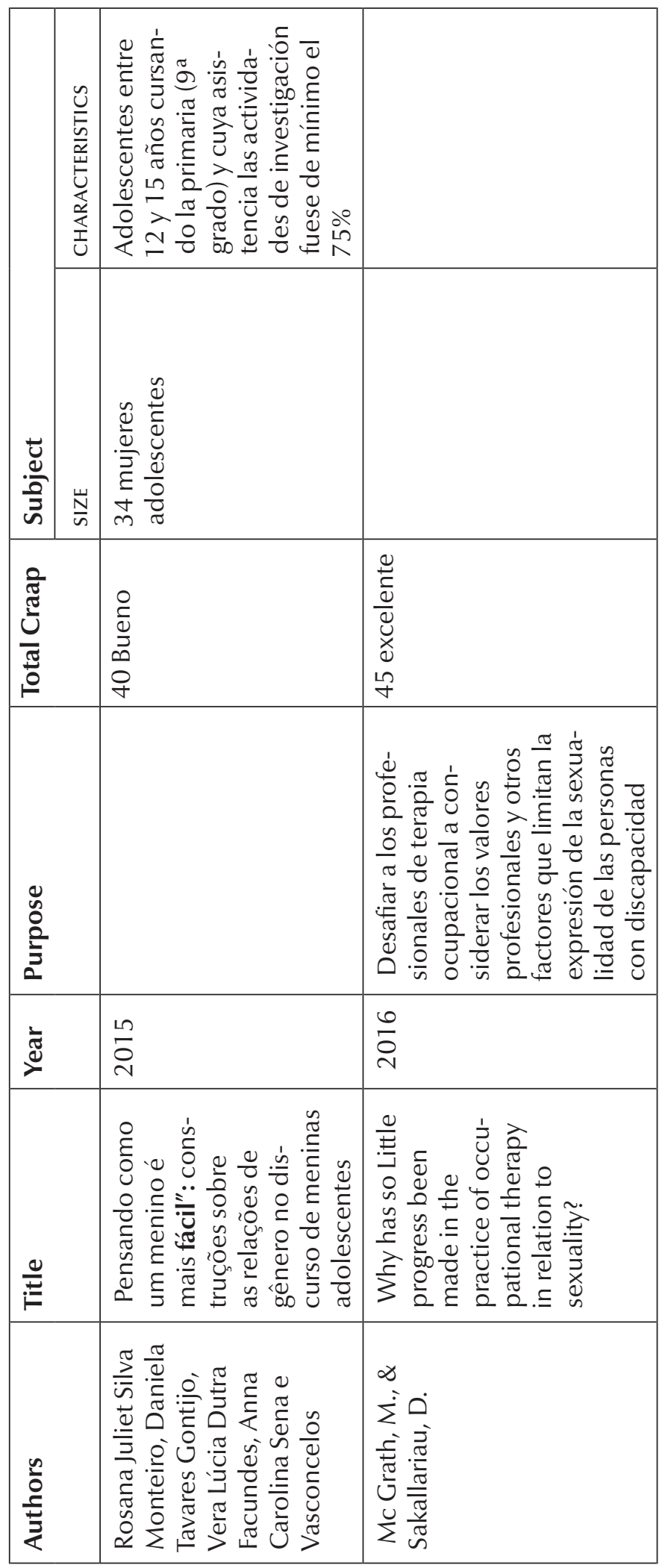


